\title{
La estimación del riesgo cardiovascular mediante puntajes mejoró el perfil de riesgo pero no redujo la incidencia de eventos
}

The estimation of cardiovascular risk through scores improved the risk profile but did not reduce the incidence of events

\section{Objetivos}

Evaluar la efectividad de los puntajes de riesgo cardiovascular (PRCV) como herramienta de prevención cardiovascular primaria, en términos de la evolución de los factores de riesgo, la prescripción de medicación preventiva y la adopción de conductas saludables.

Fuentes de datos, selección de estudios y extracción de datos Cochrane Central Register of Controlled Trials, MEDLINE, EMBASE, Conference Proceedings Citation Index-Science (CPCl-
Karmali K y col. Cochrane Database Syst Rev. 2017, Issue 3. CD006887

S), ClinicalTrials.gov, WHO International Clinical Trials Registry Platform (ICTRP), hasta marzo de 2016. Tres revisores independientes seleccionaron 41 ensayos aleatorizados y controlados $(n=194.035)$ que habían comparado la utilización de PRCV como parte de una intervención preventiva en personas sin enfermedad cardiovascular establecida versus "cuidados usuales" sin utilización de PRCV.

\section{Resultados principales}

Los resultados se resumen en la tabla 1.

Tabla 1. Efectividad del uso de puntajes de riesgo cardiovascular en prevención primaria.

\begin{tabular}{|c|c|c|c|}
\hline \multicolumn{2}{|c|}{ Principales parámetros evaluados } & Número de ensayos y participantes & Efectividad del uso puntajes de riesgo cardiovascular (IC 95\%) \\
\hline \multicolumn{2}{|c|}{ Eventos cardiovasculares } & $3 / 99.070$ & $\operatorname{RR} 1,01(0,95$ a 1,08$)$ \\
\hline \multicolumn{2}{|l|}{ Colesterol total } & $12 / 20.437$ & $\mathrm{DM}-0,10 \mathrm{mmol} / \mathrm{L}(-0,20 \mathrm{a} 0)$ \\
\hline \multirow{2}{*}{$\begin{array}{l}\text { Presión arterial } \\
\text { sistólica }\end{array}$} & Sistólica & $16 / 32.954$ & DM $-2,77$ mmHg $(-4,16$ a $-1,38)$ \\
\hline & Diastólica & $14 / 22.378$ & $\mathrm{DM}-1,12 \mathrm{mmHg}(-2,11$ a $-0,13)$ \\
\hline \multicolumn{2}{|c|}{ Cambio en el riesgo cardiovascular } & $9 / 9.549$ & DME $-0.21(-0,39$ a $-0,02)$ \\
\hline
\end{tabular}

${ }^{\star} \mathrm{RR}$ : riesgo relativo. DM: diferencia de medias. DME: Diferencia de medias estandarizada.

De acuerdo a la clasificación GRADE (en inglés: Grades of recommendation, assessment, development, and evaluation), la calidad de la evidencia identificada fue baja.

\section{Conclusiones}

La utilización de PRCV en prevención primaria no logró disminuir la incidencia de eventos cardiovasculares pero logró reducir levemente el riesgo global y los factores de riesgo individuales como el colesterol total y los niveles de presión arterial. También condujo a un incremento de las dosis o a una nueva prescripción de medicación antilipídica, antihipertensiva o antiagregante en pacientes de alto riesgo (definidos como aquellos con riesgo mayor a $10 \%$ a diez años o con diabetes).

Fuente de financiamiento / conflicto de intereses

Northwestern University, National Heart, Lung, and Blood Institute (EE. UU.), National Institute for Health Research (Reino Unido). Algunos autores de la revisión tienen relación con empresas farmacéuticas.

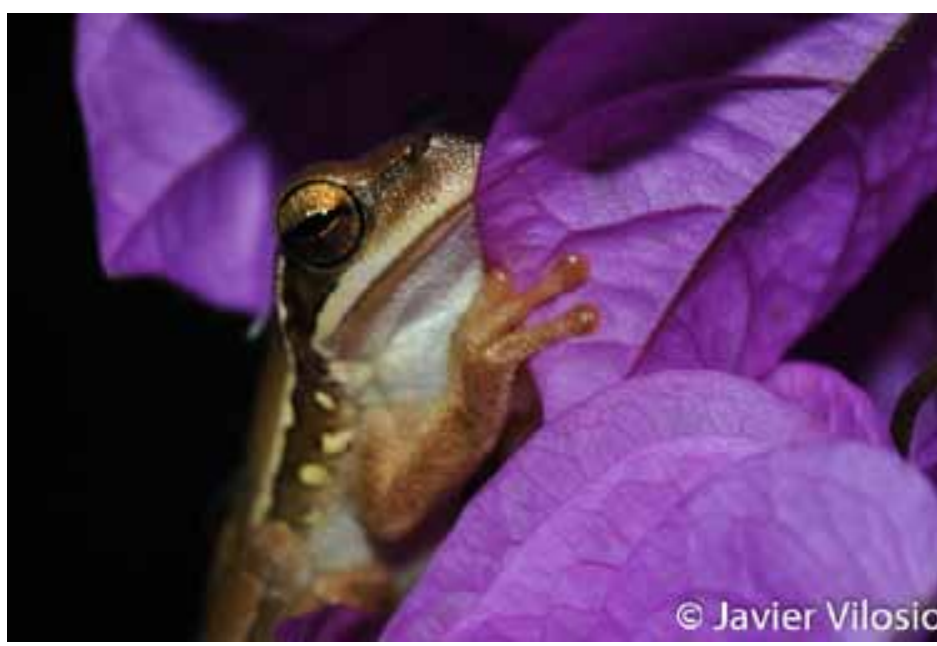

Cortesía de Javier Vilosio 


\section{Comentario}

La naturaleza multifactorial de la enfermedad cardiovascular llevó al desarrollo y utilización de PRCV basados en la valoración de determinados factores de riesgo. Estos puntajes estiman el riesgo absoluto de padecer un primer evento cardiovascular durante cierta ventana temporal y, de esta manera, pueden ayudar a definir las medidas preventivas adecuadas. También representan una valiosa herramienta para motivar a los pacientes a realizar cambios en su estilo de vida. El primer PRCV fue el de Framingham (1998, con versiones posteriores en 2002 y 2008) al que le siguieron muchos otros de acuerdo a la realidad epidemiológica de cada país o región. Estos PRCV han sido incorporados en muchas guías de prevención cardiovascular (NCEP 2002, NICE 2014, WHO 2007, ACC-AHA 2013). Sin embargo, a pesar de la extendida recomendación para utilizarlos, son inciertos sus reales beneficios en cuanto al pronóstico cardiovascular. Esta última cuestión motivó la realización de esta revisión sistemática que incluyó 41 ensayos aleatorizados y controlados que incluyó un total de 194.035 participantes que fueron sometidos a intervenciones preventivas muy variadas, desde el simple cálculo del riesgo, hasta complejas estrategias educativas (material por medios impresos y/o electrónicos, reuniones periódicas con médicos, etc.) en las que la estimación de dicho riesgo fue un componente más. El grupo control recibió información general sobre estrategias de prevención cardiovascular ("cuidados usuales"), sin utilizar PRCV.
Así mismo, hubo una gran heterogeneidad en las características de los participantes, tipos de PRCV utilizados (Framingham en 24 de los 41), duración de los estudios, etc. y por lo tanto, también, en los resultados obtenidos.

Otro problema de la revisión fue el alto riesgo de sesgo en la mayoría de los ensayos incluidos, por ejemplo el vinculado a la ausencia de cegamiento de los participantes e investigadores inherente a la naturaleza de la intervención. Por lo tanto, de acuerdo al sistema GRADE, la gran heterogeneidad y el alto riesgo de sesgo en los ensayos determinaron una baja calidad de la evidencia en cada una de las conclusiones de la revisión, Sin embargo, los resultados de este estudio son consistentes con revisiones sistemáticas previas como las de Brindle ${ }^{[1]}$ (2006), Beswick $^{[2]}(2008)$, Sheridan ${ }^{[3,4]}(2008,2010)$ y Usher-Smith ${ }^{[5]}$ (2015) en cuanto a la dificultad para documentar una mejoría del pronóstico cardiovascular con la utilización de PRCV.

\section{Conclusiones del comentador}

En concordancia con revisiones previas, este estudio no logro documentar que el uso de PRCV mejore el pronóstico cardiovascular, más allá de una leve mejoría en el control de los factores de riesgo. Sin embargo, dado que la calidad de la evidencia es baja, éstos resultados deberían ser interpretados con precaución.

Sergio Carlos Kolinski [ Servicio de Medicina Familiar y Comunitaria del Hospital Italiano de Buenos Aires. sergio.kolinski@ hospitalitaliano.org.ar ]

Kolinski S. La estimación del riesgo cardiovascular mediante puntajes mejoró el perfil de riesgo pero no redujo la incidencia de eventos. Evid Act Pract Ambul. 2017;20(4): 92-93. Comentado de: Karmali K y col. Risk scoring for the primary prevention of cardiovascular disease. Cochrane Database of Systematic Reviews 2017, Issue 3. Art. No: CD006887.

\section{Referencias bibliográficas}

1. Brindle P y col. Accuracy and impact of risk assessment in the primary prevention of cardiovascular disease: a systematic review. Heart. 2006;92(12):1752-9. https://www.ncbi. nlm.nih.gov/pubmed/16621883

2. Beswick A y col. A Systematic Review of Risk Scoring Methods and Clinical Decision Aids Used in the Primary Prevention of Coronary Heart Disease (Supplement) [Internet] A Systematic Review of Risk Scoring Methods and Clinical Decision Aids Used in the Primary Prevention of Coronary Heart Disease (Supplement). Royal College of General Practitioners (UK); 2008. https://www.ncbi.nlm.nih.gov/pubmed/21834196

3. Sheridan SL, Crespo E. Does the routine use of global coronary heart disease risk scores translate into clinical benefits or harms? A systematic review of the literature. BMC Health Serv Res. 2008:8. https://www.ncbi.nlm.nih.gov/pubmed/18366711

4. Sheridan SL y col. The effect of giving global coronary risk information to adults: A systematic review. Vol. 170, Archives of Internal Medicine. 2010. p. 230-9. https://www.ncbi. 4. Sheridan SL y col. The effect of giving global coronary risk information to adults: A systematic review. Vol. 170, Archives of Internal Medicine. 2010. p. 230-9. https://www.ncbi.
nlm.nih.gov/pubmed/20142567

5. Usher-Smith JA y col. Impact of provision of cardiovascular disease risk estimates to healthcare professionals and patients: a systematic review. BMJ Open. 2015;5(10):e008717. https://www.ncbi.nlm.nih.gov/pubmed/26503388 